\title{
Expression of concern from the Editor-in-Chief
}

It has been brought to my attention that Tong's article "Exact solutions for the flow of non-Newtonian fluid with fractional derivative in an annular pipe" published in the journal Science in China Series G: Physics, Mechanics \& Astronomy in 2005 [1] contains, in part, similar information to Tong's earlier published paper "Exact solutions for the unsteady rotational flow of non-Newtonian fluid in an annular pipe" published in the journal International Journal of Engineering Science in 2005 [2].

Science in China Series G: Physics, Mechanics \& Astronomy participates in the Committee on Publication Ethics (COPE, $\mathrm{http}: / /$ publicationethics.org/) and acts in accordance with their guidelines relating to the integrity of the work submitted to, or published in, the journal.

Following the COPE guidelines with regard to suspected redundant publication in a published article, I have examined the two articles and identified partial overlap.

Both of the two articles studied the flows of an Oldroyd-B fluid in an annular pipe, but article [1] focused on the unidirectional transient flows, while article [2] investigated the rotational flows. The two questions studied are similar with each other in mathematical constitutions, therefore similar constitutive models, basic equations, boundary and initial conditions, and transform were adopted in the solving process too. As the content and results of article [2] served as a significant foundation of article [1] which was published a few months later, article [1] should actually be considered as an extension of article [2]. However, the authors did not cite article [2] when writing article [1], and did not point out the heredity and relationship between these two articles. They also did not delete the identical part of the two articles or simplify the similar part. This makes a wrong impression that article [1] is a new research paper reporting on original new results and absolutely independent of article [2]. This should be considered a misconduct which violates the relevant guideline of the COPE. Unfortunately, my journal did not become aware of the publication of the article [2] while article [1] was in its final editing stage. We apologize to the readers for this oversight and any inconvenience caused due to insufficient information provided by the authors.

This expression of concern from Editor-in-Chief is to clarify the position of SCIENCE CHINA Physics, Mechanics \& Astronomy (formerly Science in China Series G: Physics, Mechanics \& Astronomy), as well as to thank the careful reader of my journal who spotted the overlap between the two articles.

Prof. WANG DingSheng Editor-in-Chief

SCIENCE CHINA Physics, Mechanics \& Astronomy

1 Tong D K, Wang R H, Yang H S. Exact solutions for the flow of non-Newtonian fluid with fractional derivative in an annular pipe. Sci China Ser G-Phys Mech Astron, 2005, 48(4): 485-495

2 Tong D K, Liu Y S. Exact solutions for the unsteady rotational flow of non-Newtonian fluid in an annular pipe. Int J Eng Sci, 2005, 43: 281-289 